\title{
NNARX Networks on Didactic Level System Identification
}

\author{
A. F. SANTOS NETO \\ CEFET-MG \\ Department of Eletroelectronics \\ José Peres Street, 558, Leopoldina \\ BRAZIL \\ V. F. VIDAL \\ UFJF \\ Department of Energy Systems \\ José Lourenço Kelmer Street, Juiz de Fora \\ BRAZIL
}

M. F. SANTOS

CEFET-MG

Department of Eletroelectronics

José Peres Street, 558, Leopoldina José Lourenço Kelmer Street, Juiz de Fora

BRAZIL
A. C. SANTIAGO
UFJF

Department of Energy Systems

BRAZIL

\author{
P. MERCORELLI \\ Leuphana University of Lüneburg \\ Institute of Product and Process Innovation \\ D-21335, Lüneburg \\ GERMANY
}

\begin{abstract}
This work has as main objective to propose the identification of a small scale non-linear system through the Neural Network AutoRegressive with eXternal input. The use of this network requires an adequate methodology for its configuration and, consequently, a good training set. Then, it is proposed that the main definitions of the network parameters be obtained through the analysis of nonintrusive performance indices. Additionally, using a database based on the system's response, excited by the Pseudo-Random Binary Sequence signal. The methodology will be applied in two specific open-loop identification situations: numerical simulation of a fourth order polynomial system (Case 01), and an experimental system that controls a nonlinear water tank level (Case 02). The results of the identified models were able to represent the system dynamics with high fidelity, presenting an average identification error of less than $\mathbf{0 . 1 4}$ and $\mathbf{0 . 3 4 \%}$ for Case 1 and 2, respectively. Also, it is observed that the learning and generalization evidence could represent the process intrinsic nonlinearities satisfactorily. Besides, it willbepossibletofindthepotentialityandusefulnessofthedevelopednetworkinnonlinearsystemidentification.
\end{abstract}

Key-Words: NNARX, System Identification, Level System.

Received: November 2, 2019. Revised: April 1, 2020. Re-revised: April 18, 2020. Accepted: April 30, 2020. Published: May $12,2020$.

\section{Introduction}

It is known that system mathematical modelling has great importance in the treatment of various engineering problems. With "good mathematical models", it is possible to get a reliable system behavior and design suitable controllers to the issue under study, reducing the costs through simulation benefits, prototyping, among other advantages [1].

The search for accurate models implies to consider the system nonlinear characteristics, such as transport delays, saturation, and parameters variation/sensitivity. In these situations, it could be said that the application of linear modelling techniques may not be adequate, since they can not represent certain system complexities [1,2].

For the solution of this challenge, many works were developed in the system identification area, treating the problem with neural networks and other approaches [3, 4].

As recent examples of Neural Network AutoRe- gressive with eXternal input (NNARX) application, some related works may be mentioned. The work [5] shows the application of the NNARX model and its comparison with traditional models (gray-box and black-box) to simulate internal temperatures of a commercial building in Montreal (QC, Canada). Results show that the neural networks mimic more accurately the thermal behavior of the building, compared to gray-box and black-box linear models, in some scenarios.

In [6], the model of a Direct Current (DC) motor is identified using an algorithm developed in MATLAB/SIMULINK platform. The simulation tests showed good results from the network learning and good accuracy in the system dynamics description.

Work [7] presents an application in agriculture, specifically to improve environmental conditions and increase the fruits and vegetables shelf life. The NNARX was developed for predicting internal temperature and relative humidity of an evaporation cooler. The resulting models driven by the Levenberg- 
Marquardt back propagation algorithm showed high performance for the cooler internal variables prediction, which makes it possible to avoid the loss of the products a few days after the harvest.

The authors from work [8] use the NNARX to identify and model a hydro-turbine generating. The random guide vain signal is used to train NNARX and an improved Levenberg-Marquardt algorithm is proposed in this article. Simulation results indicate that NNARX model with improved L-M algorithm can reach high recognition accuracy and have good generalization ability. More important works can be highlighted, such as $[9,10,11,12]$.

Among several implicit advantages in the mentioned works, this approach allows to describe nonlinear systems considering linear difference equations, taking the current output for previous inputs and outputs as parameters. It is suitable for modelling both stochastic and deterministic system components and can describe a nonlinear system variety [13].

In this direction, this work presents the identification of a small-scale nonlinear system used in the Laboratory of Automatic Control in CEFET-MG (Campus Leopoldina). The main contribution of this article is based on the configuration of the NNARX methodology, whose number of hidden layers and neurons will be defined through the analysis of nonintrusive performance indices. Another contribution is the method application in a typical process control problem using the Pseudo-Random Binary Sequence (PRBS) signal, as in the input signal test. This signal type has the excitation persistent suit to the nonlinear system, as present the literature [4].

The methodology was applied in two specific open-loop identifications: numerical simulation of a fourth order system and the experimental prototype of a water level tank control with nonlinear characteristics. Then, this work will be also able to observe the evidence of learning and generalization, allowing to represent the natural process nonlinearities.

This paper is organized as follows: Section 2 presents the prototype developed to perform the techniques proposed hereafter; Section 3 describes the NNARX basic concepts; Section 4 deals with the simulated and experimental results. Finally, Section 5 presents the final considerations and conclusions.

\section{Level Tank System Designed}

The system designed to apply the methodology consists of a small-scale tank system submitted to level control. The system in Fig. 1 is currently one of the prototypes used in the Laboratory of Automatic Control in CEFET-MG (Campus Leopoldina) by un- dergraduate students in Control and Automation Engineering.

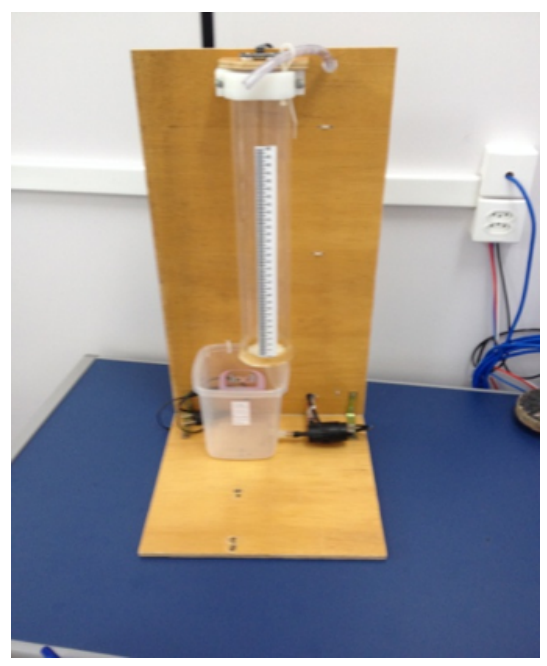

Figure 1: Level control plant used in the experimental tests

The plant, in principle, is composed of four subsystems: measurement, actuation, control and physical structure. The measuring system is composed of: Ultrasonic sensor, responsible for measuring the cylindrical reservoir level and sending it to the controller; Actuator, composed of an electro-pump, a power supply, an electronic circuit of the interface between the actuator and the controller; Control system composed of an Arduino UNO board, responsible for the intervention in the plant through the message treatment sent by the measurement system and subsequent perform them on the actuators; Physical structure, consisting of a central (cylindrical) tank and an auxiliary tank, among other additional components. The main components used for the system assembly are shown in Table 1.

Table 1: Main components of level control plant used in one experiment

\begin{tabular}{ll}
\hline Component & Specification \\
\hline Measurement & HC-SR04 Ultrasonic \\
. Actuator & $12 V_{D C}$ Pump (60W) \\
Controller & Arduino UNO \\
Power Supply & DC Source (12V/10A) \\
\hline
\end{tabular}

\section{NNARX Model Identification}

The NNARX consists of a neural network based on a specific structure of linear identification. Among the 
existing structures, all are structured in the vector of auto regression, i. e., they use previous values of the system excitation input signal to estimate the current system output $[14,15]$. In this context, it is possible to cite: Finite Impulse Response (FIR), AutoRegressive eXogenous input (ARX), AutoRegressive Moving Average with Xogenous inputs (ARMAX), Output Error (OE) and State Space Innovations Form (SSIF) [14].

The NNARX structure arises from the neural model reconciliation with the ARX regression vector choice. It should be emphasized that the variables measured values of the process output are added to the regression vector. Mathematically the expression of extended nonlinear model of an NNARX is described as (1):

$$
\begin{array}{r}
y^{\prime}(k)=f(y(k-1), \ldots, y(k-n), \\
u(k-d), \ldots, u(k-d-m))
\end{array}
$$

where: $y$ and $u$ are the output and input plant signals, respectively; $y^{\prime}$ is the estimated plant output; $k$ is the current instant; $d$ is the plant delay time; $m$ is the number of input delays and $n$ is the number of output delay; $f(\cdot)$ is the nonlinear function mapped by the Artificial Neural Network (ANN).

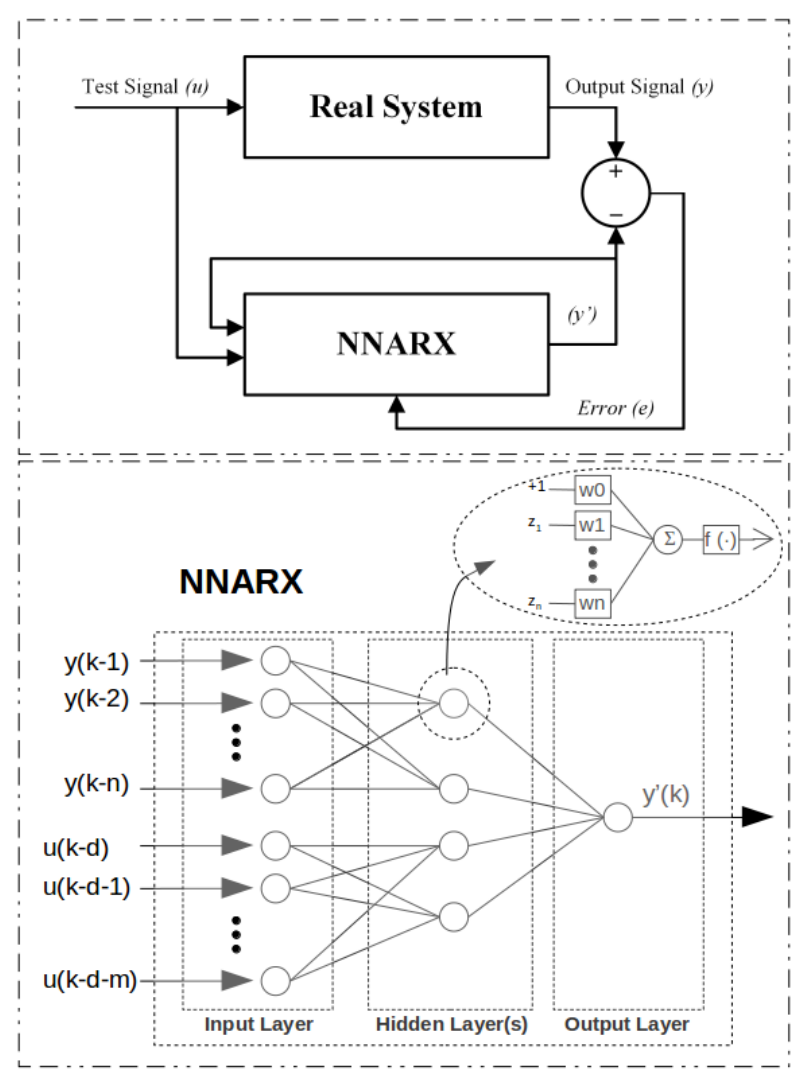

Figure 2: NNARX illustration.
Also, the ANN used to map the function $f(\cdot)$ is conceptually a multilayer perceptron with the following characteristics: input layer as source nodes; hidden layer with sigmoid activation function; output layer with only 1 neuron and linear activation function [16].

In the following topics, the methodology used for the network setting will be described. That is, how the number of hidden layers and their respective number of neurons and others parameters were chosen.

\subsection{NNARX Application Methodology}

The procedure below was developed to apply and identify the system model, comprising:

- Choice of Excitation Signal: a fundamental aspect for the system identification involves the choice of excitation signals. A proper choice allows static and dynamic characteristics to be identified [4]. If they are not excited, such information can not be identified and represented by the model. Among the excitation signals, the literature recommends the use of PRBS or even random signals [17]. In this work, only the PRBS signals are regarded.

- ANN configuration and training: this important stage consists of the network structure definition, that is, the number of network inputs, hidden layers and their subsequent training stage. In this work, the methodology proposes to define the number of hidden layers and neurons of each layer by nonintrusive performance indices analysis, using the Integral of the Absolute Error (IAE) or Integral of the Squared Error (ISE). The definition of the number of layers and neurons is done by analyzing the effect of setting the number of neurons and hidden layers. After analyzing the tested configurations, the network configuration is defined by the arrangement with the lowest indices ac iae or ac ise. This process can be seen in algorithm 1: 


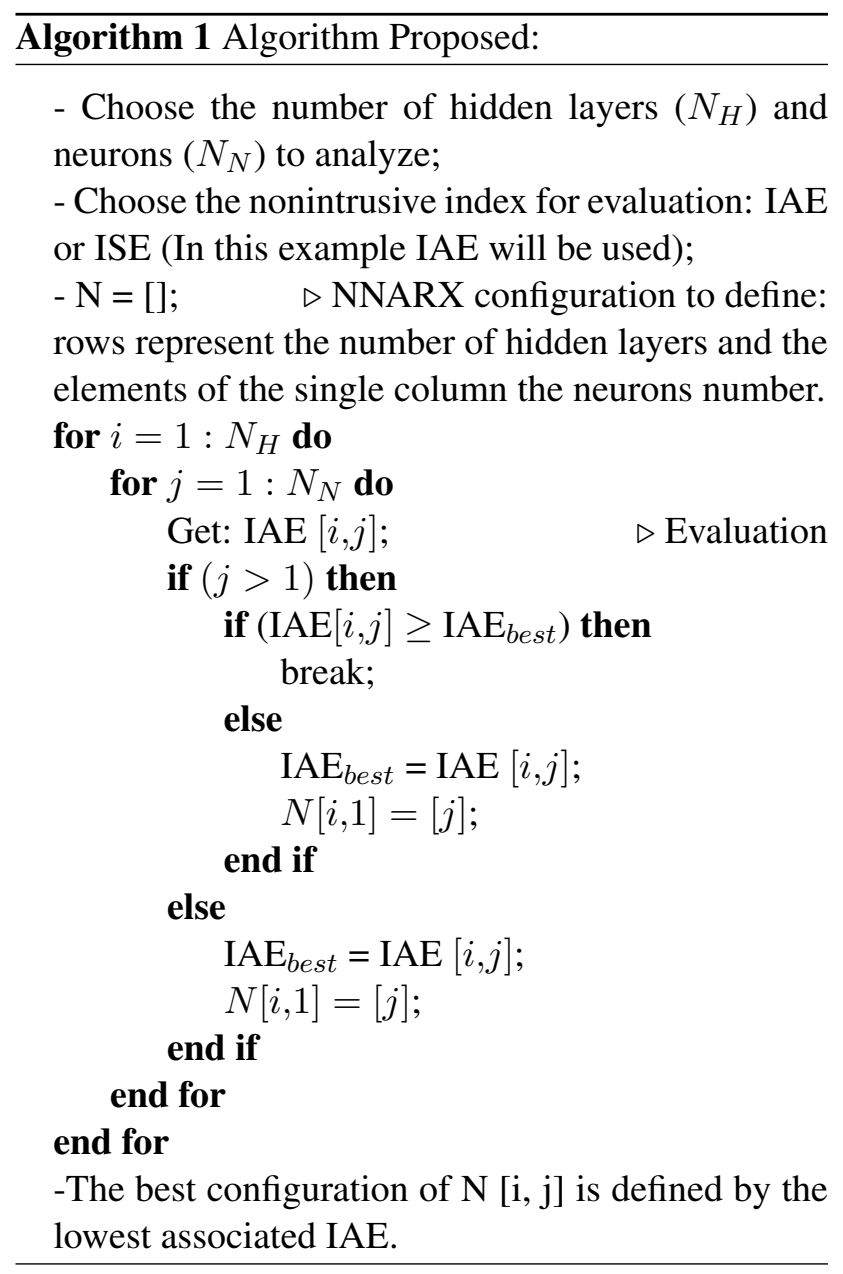

Since the training process has a random character in the network weight initialization, it was decided to analyze 10 events for each distinct network configuration to define the network with greater reliability.

Additionally, the back-propagation algorithm via Levenberg-Marquardt was used as an alternative to perform network parameter adjustments, based on the Mean Squared Error (MSE) gradient; also, a toolbox of the MATLAB software was used for the neural network implementation; it is important to highlight that the training technique was a parallel architecture, proving to be more efficient than the serial-parallel one.

- Model Validation: stage responsible for the learning/generalization analysis of the trained network. Its behavior is analysed once submitted to different inputs from those presented for training. Finally, if the model satisfies the identification purposes, the resulting network is accepted and ready to be used; otherwise, it returns to the previous steps until it satisfies the desired goals.

\section{Simulated and Experimental Re- sults}

To identify nonlinear systems through the developed methodology, two cases were studied: the first case consists of the numerical simulation of a fourth order polynomial system with complex dynamics, represented in the following equation:

$$
G(s)=\frac{0.1}{(s+1)^{2}(s+0.4)^{2}}
$$

The second case is a real experimental system of a level control plant (Fig. 1). The goal here is to model the water reservoir level with intrinsic nonlinear characteristics (transport delay time, actuator saturation, gravity, among others). In addition, all networks were configured with: the plant delay time $d=1$; number of input delays and the output $m=3$ e $n=4$, respectively; training with 100 epochs and the data divided into subsets of training, testing, validation with the percentage of $60 \%, 30$ and $10 \%$, respectively. These definitions were made after some empirical tests.

The results followed the respective sequence: Presentation of the excitation signal; Analysis of the neurons and hidden layer numbers; Definition of the number of hidden layer neurons.

\subsection{Case $01-4^{\text {th }}$ Order System}

Excitation signal and response system: using the MATLAB software, the following PRBS signal and plant response were generated and presented in Fig. 3.

Analysis of the hidden layer and neuron number: the number of neurons in the hidden layer was evaluated incrementally and analyzed through the performance of the NNARX during the training phase, once submitted to the signal presented in Fig. 4. Additionally, it is important to point out that the result of the second hidden layer onward adds the best result of the previous layer.

Definition of NNARX setting: from the results obtained in Fig. 4 and Table 2, it can be seen that increasing the number of neurons does not necessarily represent greater learning ability. Therefore, this methodology defines the neural network with: 1 neuron in the first hidden layer. 

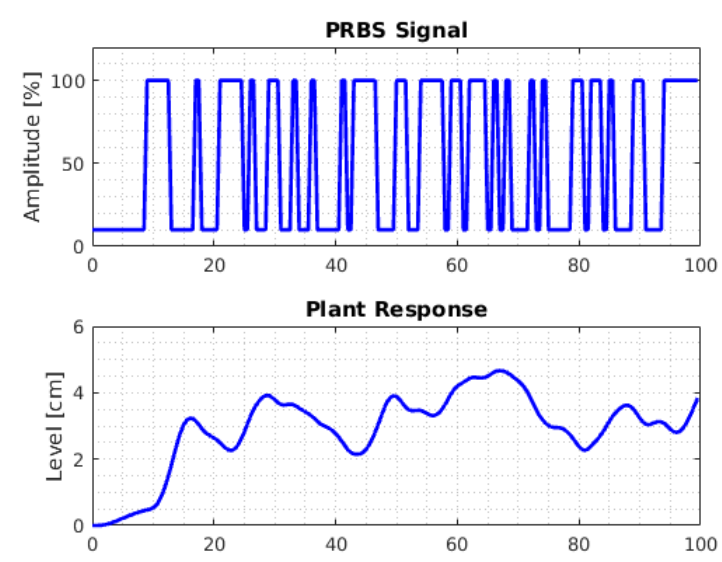

(a) System excitation signal and plant response.
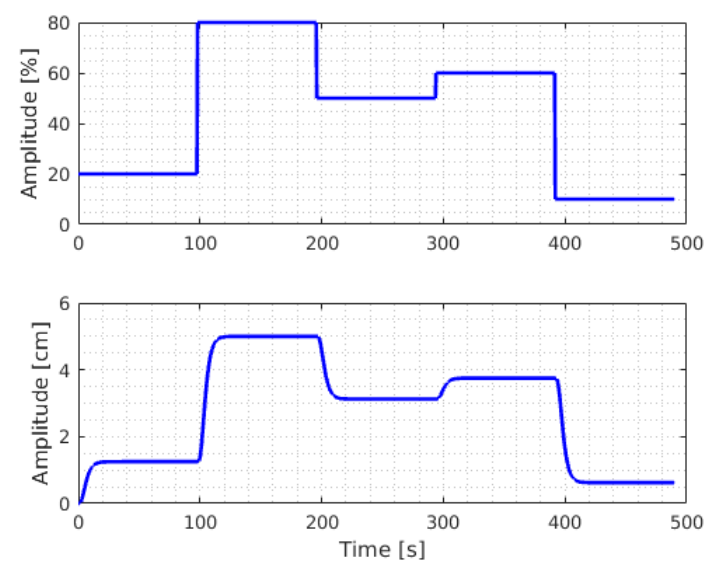

(b) Validation data of Case 01.

Figure 3: System excitation signal and validation data of Case 01.

Table 2: Main results of Case 01.

\begin{tabular}{lccc}
\hline Number of layers & Best result & IAE & ISE \\
\hline $1^{\text {st }}$ Hidden Layer & 1 Neuron & 09.9248 & 0.2557 \\
$2^{s t}$ Hidden Layer & 2 Neurons & 14.5735 & 0.3518 \\
$3^{s t}$ Hidden Layer & 2 Neurons & 27.1077 & 1.3301 \\
\hline
\end{tabular}

\subsection{Case 02 - Physical Experimental System}

Excitation signal and system response: in this case, the following PRBS signal was also generated using the MATLAB software and embedded in Arduino platform to obtain the plant response presented in Fig. 5.

Analysis of the hidden layer and neuron numbers: as in Case 01, the number of neurons and hidden layers will be analyzed through the training and valida-
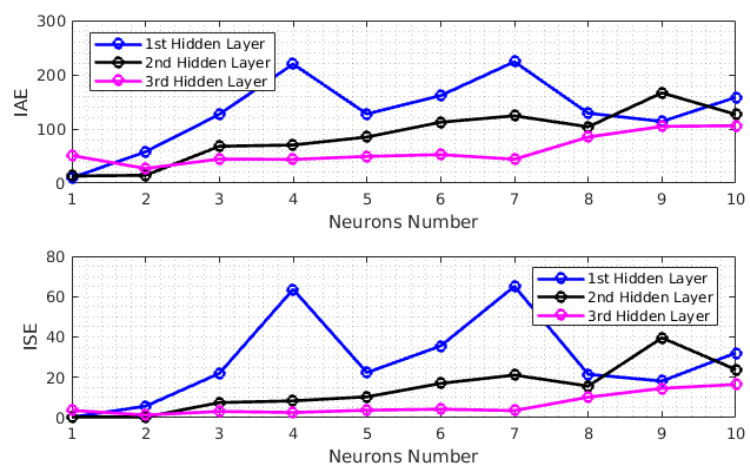

(a) Results of the network configuration analysis.
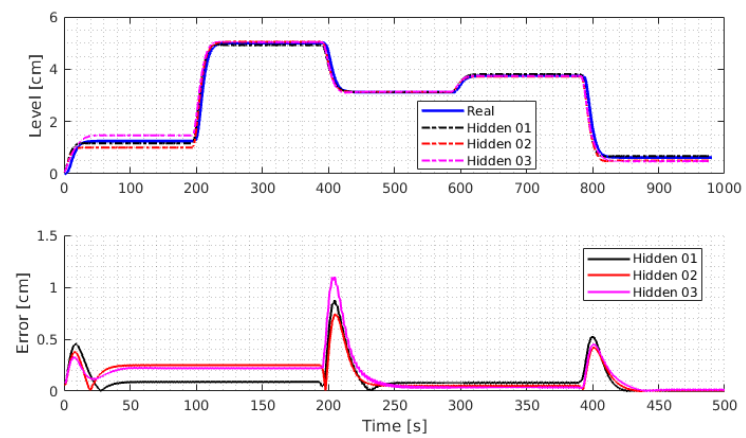

(b) Results of Case 01 .

Figure 4: Results of NNARX analysis and results of Case 01 .

tion data, presented in Fig. 6. It is worth mentioning that the system sampling time is $500 \mathrm{~ms}$.

From the obtained results, it can be seen in Fig. 6 and Table 3 that increasing the number of neurons represents a learning ability increased to the first layer. However, the insertion of the third layer did not perform better learning.

Table 3: Main results for Case 02.

\begin{tabular}{lccc}
\hline Number of layers & Best result & IAE & ISE \\
\hline $1^{\text {st }}$ Hidden Layer & 3 Neurons & 24.9585 & 1.9871 \\
$2^{\text {nd }}$ Hidden Layer & 2 Neurons & 24.6481 & 1.8806 \\
$3^{r d}$ Hidden Layer & 1 Neuron & 31.2248 & 2.9493 \\
\hline
\end{tabular}

Definition of NNARX setting: as can be understood from Table 3, the third hidden layer does not represent a significant improvement over the established 2 hidden layers. Therefore, this methodology defines the neural network with 2 hidden layers: 3 neurons in the first and 2 neurons in the second one. 

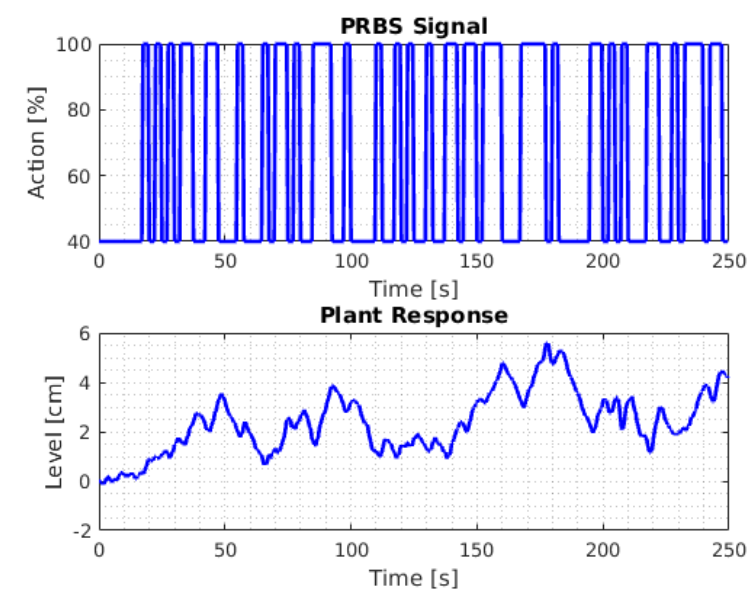

(a) System excitation signal and plant response.
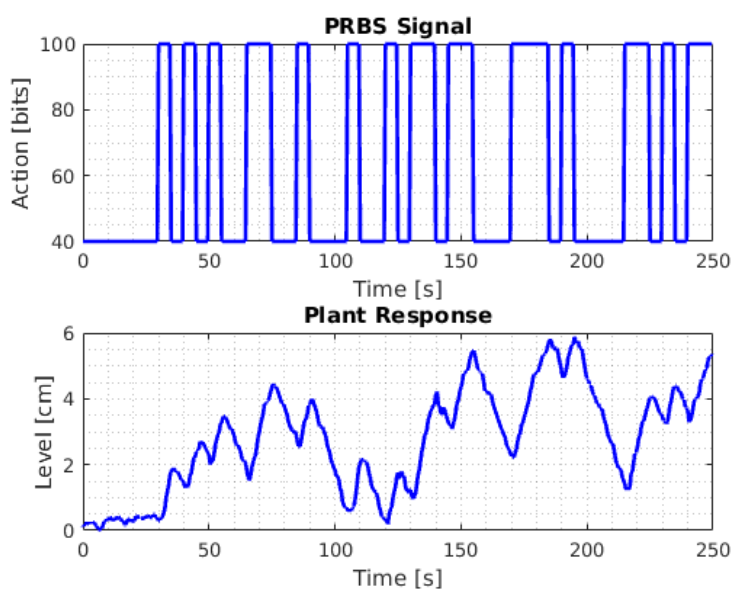

(b) Validation results of Case 02.

Figure 5: Analysis of the system excitation signal and validation data of Case 02 .

\section{Conclusions}

Given the presented results, it is possible to observe the evident learning and generalization capacity of the developed NNARX. In both cases, the networks were able to identify the system behavior with mean errors of less than $0.02 \mathrm{~cm}$, or $0.14 \%$ (Case 01 ), and 0.05 $\mathrm{cm}$, or $0.34 \%$ (Case 02 ), in a scale from 0 to $15 \mathrm{~cm}$. That is, errors smaller than the accuracy of the $0.4 \mathrm{~cm}$ ultrasonic sensor, which shows that the network was successful in these studies.

Finally, it is possible to state that the methodology for the adjustment and application of NNARX had interesting results, getting represent satisfactorily the process intrinsic nonlinearities. Fact that indicates its potential and possible use of the network developed in the identification of other similar non-linear systems.
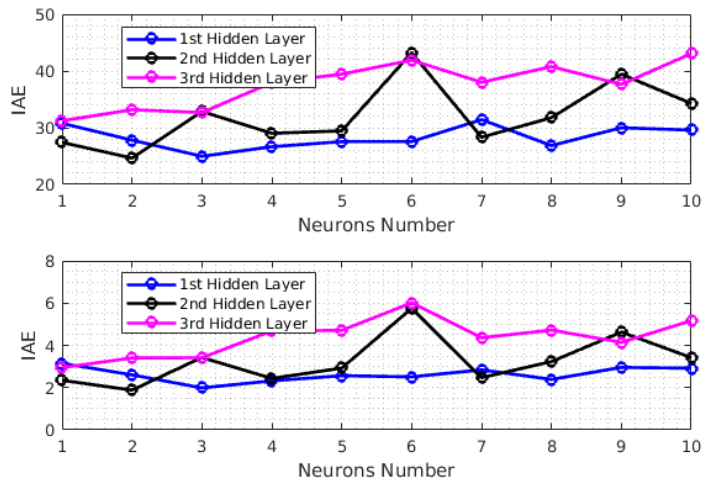

(a) Results of the network configuration analysis.
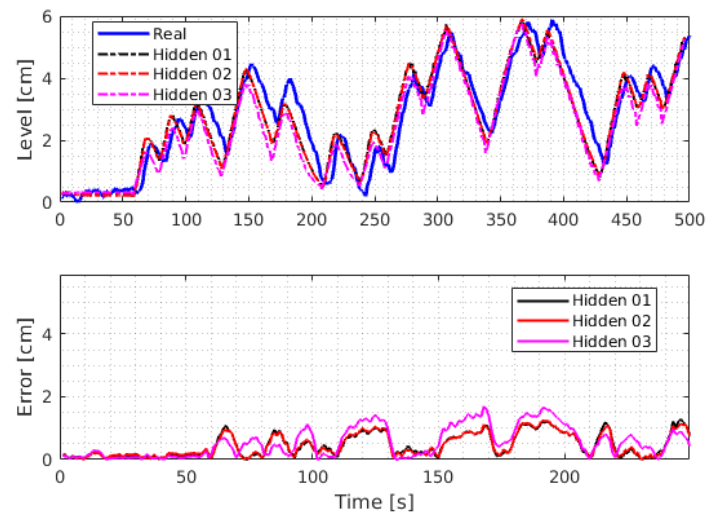

(b) Results of Case 02.

Figure 6: Validation data and results of Case 02.

\subsection{Future Works}

The conclusion of this work opens some future works, such as test the methodology in different plants, and verifies its applicability, comparing the proposed methodology with other traditional identification techniques and neural network topologies.

\section{Acknowledgment}

The authors would like to thank CEFET-MG and Leuphana University of Lüneburg for the financial support.

\section{References:}

[1] L. Ljung, Identification of nonlinear systems. Linköping University Electronic Press, 2007.

[2] O. Nelles, Nonlinear system identification: from classical approaches to neural networks and fuzzy models. Springer Science \& Business Media, 2013. 
[3] L. Ljung, "Perspectives on system identification," Annual Reviews in Control, vol. 34, no. 1, pp. 1-12, 2010.

[4] R. Isermann and M. Münchhof, Identification of dynamic systems: an introduction with applications. Springer Science \& Business Media, 2010.

[5] B. Delcroix, J. Le Ny, M. Bernier, M. Azam, B. Qu, and J.-S. Venne, "Autoregressive neural networks with exogenous variables for indoor temperature prediction in buildings," in Building Simulation, pp. 1-14, Springer, 2020.

[6] Y. Naung, A. Schagin, H. L. Oo, K. Z. Ye, and Z. M. Khaing, "Implementation of data driven control system of dc motor by using system identification process," in 2018 IEEE Conference of Russian Young Researchers in Electrical and Electronic Engineering (EIConRus), pp. 1801-1804, IEEE, 2018.

[7] I. Sow, E. Murimi, and U. Mutwiwa, "Evaporative cooler climate prediction using artificial neural network," JOURNAL OF SUSTAINABLE RESEARCH IN ENGINEERING, vol. 5, no. 3, pp. 113-127, 2020.

[8] Z. H. Xiao, Z. P. An, S. Q. Wang, and S. Q. Zeng, "Research on the nnarx model identification of hydroelectric unit based on improved Im algorithm," in Advanced Materials Research, vol. 871, pp. 304-309, Trans Tech Publ, 2014.

[9] M. Rajalakshmi, G. Saravanakumar, and C. Karthik, "Nonlinear identification of ph processes by using nnarx model," in Proceedings of the International Conference on Computing and Control Engineering (ICCCE'12), 2012.

[10] R. Adnan, A. M. Samad, Z. M. Zain, and F. A. Ruslan, "5 hours flood prediction modeling using improved nnarx structure: case study kuala lumpur," in 2014 IEEE 4th International Conference on System Engineering and Technology (ICSET), vol. 4, pp. 1-5, IEEE, 2014.
[11] T. Mulyana, "Nnarx model structure for the purposes of controller design and optimization of heat exchanger process control training system operation," in AIP Conference Proceedings, vol. 1831, p. 020040, AIP Publishing LLC, 2017.

[12] F. A. Ruslan, A. M. Samad, and R. Adnan, "Modelling of flood prediction system using hybrid nnarx and extended kalman filter," in 2017 IEEE 13th International Colloquium on Signal Processing \& its Applications (CSPA), pp. 149152, IEEE, 2017.

[13] M. H. F. Rahiman, M. N. Taib, R. Adnan, and Y. M. Salleh, "Analysis of weight decay regularisation in NNARX nonlinear identification," in 5th International Colloquium on Signal Processing Its Applications, pp. 355-361, March 2009.

[14] M. Girault, E. Videcoq, and D. Petit, "Estimation of time-varying heat sources through inversion of a low order model built with the modal identification method from in-situ temperature measurements," International Journal of Heat and Mass Transfer, vol. 53, pp. 206-219, 01 2010

[15] K. S. Narendra and K. Parthasarathy, "Identification and control of dynamical systems using neural networks," Transactions of Neural Networks, pp. 4-27, 1990.

[16] M. Norgaard, O. Ravn, N. Poulsen, and L. Hansen, Neural networks for modelling and control of dynamic systems: a practitioner's handbook. Advanced textbooks in control and signal processing. Springer, Berlin, 2000.

[17] L. A. Aguirre, Introdução à Identificação de Sistemas - Técnicas Lineares e Não-Lineares Aplicadas a Sistemas Reais. Editora UFMG, 2007. 